\title{
Supervised Associative Learning in Spiking Neural Network
}

\author{
Nooraini Yusoff and André Grüning, \\ Department of Computing, Faculty of Engineering and Physical Sciences, \\ University of Surrey, Guildford, GU2 7XH Surrey, UK \\ \{n.yusoff, a.gruning\}@surrey.ac.uk
}

\begin{abstract}
In this paper, we propose a simple supervised associative learning approach for spiking neural networks. In an excitatory-inhibitory network paradigm with Izhikevich spiking neurons, synaptic plasticity is implemented on excitatory to excitatory synapses dependent on both spike emission rates and spike timings. As results of learning, the network is able to associate not just familiar stimuli but also novel stimuli observed through synchronised activity within the same subpopulation and between two associated subpopulations.
\end{abstract}

Keywords: Spiking neural network, Associative learning, Supervised learning, Excitatory-Inhibitory network, Izhikevich spiking neurons.

\section{Introduction}

There is evidence in neurophysiology that long-term association between stimuli, which involves synaptic plasticity, is triggered by overlapping short-term activity, which only involves activity dynamics (e.g. [1], [7] and [8]), linking neuronal activity and long-term memory.

Associative-based learning can be implemented using unsupervised or supervised approaches [6]. For unsupervised learning, perhaps the temporal variant of Hebbian learning known as spike-timing dependent plasticity (STDP) is the most biologically plausible approach. However, the purely unsupervised approach is often not suitable for goal-oriented applications, so it is used in this paper with prescribed target stimuli as a form of supervision. Such supervisory signal could be assumed to come from another part of the brain [2].

In this study, we explore a supervised associative learning algorithm as a combination of spike emission rate dependent and STDP approaches from [9] on a learning task similar to [8]. Supervision in learning is only through intensified currents into paired target neuron subpopulations. Learning is performed by associating two different stimuli with synchronisation of network activity within and between subpopulations of neurons as the key measure of stimulus association. 


\section{Simulation Model}

For our simulation, the network structure is an adaptation of excitatory-inhibitory neural network model similar to [7]-[8]. The neuron model used with simple computational properties is based on the Izhikevich spiking neuron (IM) with standard parameters governing the dynamics of membrane potential (further details of the IM can be found in [3] and [4]).

The network is composed of 1000 neurons $(N=1000)$ with 800 excitatory neurons $\left(N_{E}=800\right)$ and 200 inhibitory neurons $\left(N_{I}=200\right)$. Each neuron receives synaptic contacts from $20 \%$ of excitatory neurons $\left(C_{E}=0.2 N_{E}\right)$ and $20 \%$ of inhibitory neurons $\left(C_{I}=0.2 N_{I}\right)$, randomly. The excitatory neurons population is divided into subpopulations that each represents an object for the memory under study, meanwhile the inhibitory subpopulation acts as the global network inhibition. In our simulations, there are four $(p=4, P 1-P 4)$ subpopulations of excitatory neurons with 160 (selectivity, $f=0.2 \rightarrow f N_{E}=160$ ) units each with the following allocation: P1: neurons 1-160, P2: neurons 161-320, P3: neurons 321-480 and P4: neurons 481-640, while neurons from 801-1000 are inhibitory and the remaining excitatory neurons 641-800 are the non-selective pool of neurons. The connection strengths of excitatory synapses on excitatory neurons are denoted $W_{1 / 0 / a}$, excitatory on inhibitory neurons $W_{E I}$, inhibitory on excitatory neurons $W_{I E}$, and inhibitory on inhibitory $W_{I I}$. Within $W_{1 / 0 / a}$, $W_{1}$ are the synaptic connections within the same subpopulation, $W_{a}$ is the synaptic connection between two associated subpopulations and $W_{0}$ labels the non-associated subpopulation.

\section{Learning Implementation}

For our simulation experiments, learning is performed through implementation of synaptic plasticity on excitatory to excitatory synapses $\left(W_{1 / 0 / a}\right)$. Other synapses $\left(W_{E I}\right.$, $W_{I E}$, and $W_{I I}$ ) are set to random values with moduli drawn uniformly the range from between 0 and 1 and with signs of connections depending on the type of the neuron (excitatory or inhibitory).

Learning is implemented in a Hebbian paradigm, considering both spike rate and timings of both pre-synaptic and post-synaptic neurons in a learning window [9]. In a learning trial with 500 milliseconds (ms) simulated time, the time window is divided into $100 \mathrm{~ms}(T=100)$ wide overlapping bins at $50 \mathrm{~ms}$ intervals (Fig. 1). For each learning time bin, the average spike rate of every excitatory neuron $\left(S_{\text {pre }}\right.$ and $\left.S_{\text {post }}\right)$ is estimated as the ratio of the number of spikes emitted in the bin divided by $T$ [8]. The weight adjustments, $\Delta W$ are calculated as a function of time difference, $\Delta t=t_{j}^{(f)}-t_{i}^{(f)}$, where $t_{j}^{(f)}$ and $t_{i}^{(f)}$ are the last firing times of post-synaptic neuron $j$ and pre-synaptic neuron $i$, respectively, within the learning time bin (Fig. 2) [9]. To avoid saturation of synaptic strength values infinitely, we keep the values within the range 0 to 3 . 


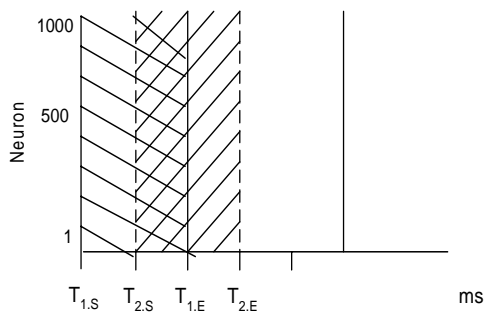

Fig. 1. Learning time bins with overlapping windows, $T_{N . S}$ is the beginning of a time bin which ends at $T_{N . . E}$ with $T_{N . . E}-T_{N . S}=100 \mathrm{~ms}$, and $T_{N . S}$ increasing in steps of $50 \mathrm{~ms}$ [8].

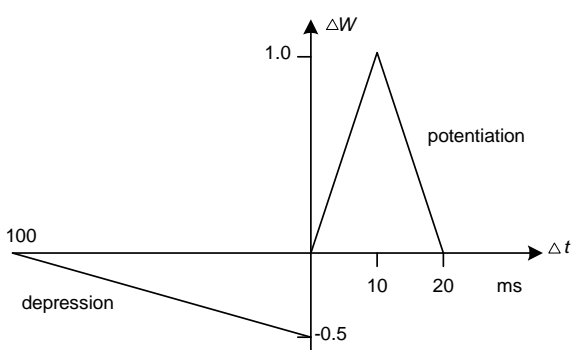

Fig. 2. A function of time difference between last firing of pre-, $t_{i}^{(f)}$, and post synaptic neuron, $t_{j}^{(f)}$, $\Delta t=t_{\text {post }}-t_{\text {pre }}=t_{j}^{(f)}-t_{i}^{(f)}$, on excitatory neurons [9], Fig. 2.

A synapse $W$ is highly potentiated (if $W(t))=0$ ) to the maximal synaptic strength $\left(w_{\max }=3\right)$ if both pre- and postsynaptic neurons emit spikes above the high rate threshold $T_{+}$and only if the time difference of the last firing between the pre- and postsynaptic neurons is above $0(\Delta t>0)$. $W$ is weakly potentiated with an amount of $\Delta W$ (derived from Fig. 2), if the pre synaptic (postsynaptic) neuron emits spikes with rate above $T_{+}$whilst the postsynaptic (pre synaptic) neuron spike emission rate is below $T_{+}$but above the low threshold, $T_{a}$. For depression of $W$, where $\Delta t<0$ from Fig. 2 , it is applied if the pre synaptic (postsynaptic) neuron emits spikes above $T_{+}$and the post-synaptic (pre synaptic) neuron emits spikes below $T_{a}$. The synaptic plasticity rules are summarised in 1-3.

$W_{i j}(t+1)= \begin{cases}w_{\max }, \quad W_{i j}(t)=0, & \left(S_{\text {pre }} \geq T_{+}, S_{\text {post }} \geq T_{+}\right), \Delta t>0 \\ \max \left(w_{\min }, \min \left(w_{\max }, W_{i j}(t)+\Delta W\right), \begin{array}{rl}{\left[\left(S_{\text {pre }} \geq T_{+}, T_{a}<S_{\text {post }}<T_{+}\right) ;\right.} \\ \left.\left(T_{a}<S_{\text {pre }}<T_{+}, S_{\text {post }} \geq T_{+}\right)\right], \Delta t>0 \\ & {\left[\left(S_{\text {pre }} \geq T_{+}, S_{\text {post }} \leq T_{a}\right) ;\right.} \\ & \left.\left(S_{\text {post }} \geq T_{+}, S_{\text {pre }} \leq T_{a}\right)\right], \Delta t<0\end{array}\right.\end{cases}$

\section{Simulation Results}

In our simulation, for every ms in each trial, each neuron receives background noisy external currents $\xi_{i}(t)$, where $\xi_{i}(t)$ is Gaussian noise with mean $\mu$ and stdev $\sigma$. Excitatory and inhibitory neurons receive external currents with standard deviations $\sigma_{N e}=3$ and $\sigma_{N i}=1$, respectively. During a learning trial, for $t>150$ to $t \leq 350 \mathrm{~ms}$, the external current distribution to target stimulus subpopulation 1 is changed to a uniform one from range 0 to $\gamma$ with $\gamma=30$. Then, for $t>250$ to $t \leq 450 \mathrm{~ms}$, the target stimulus subpopulation 2 is stimulated with the same range of currents as its subpopulation to be associated. We ran two batches of simulations: 1) learning with familiar stimuli and 2) learning with novel stimuli. For (1), a stimulus is assumed to 
have been learned prior to establishing relationship between two different stimuli, while for (2) only a small subset of synapses are initialised with some strength values.

\subsection{Learning with Familiar Stimuli}

For implementing associative learning with familiar stimulus, neurons in the same subpopulation are connected with a set of random $W_{1}$ values in the range of 0 and 3 . With such pre-initialised synaptic connections, neurons in the same subpopulation always fire synchronously. An example of associative learning results between two stimuli, P1 and P3 is depicted in Fig. 3.

A. Trial 1

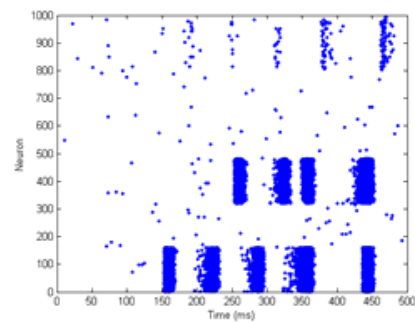

B. Trial 2

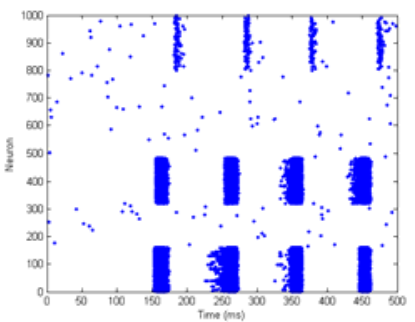

Fig. 3. Synchronisation of neuronal network activity after two learning trials for a pair of familiar stimuli P1 $\leftrightarrow$ P3. Currents to excitatory subpopulation neurons of P1 (neurons: 1-160) are intensified for $200 \mathrm{~ms}(t>150$ to $t<=350 \mathrm{~ms}$ ), then P3 (neurons: 321-480) is stimulated for the same duration $(t>250$ to $t<=450 \mathrm{~ms})$. Association of patterns is measured based on synchronous activity in each member of a stimuli learning pair subpopulation. A) Learning trial 1: activation of P1 is observed in between 350 to $450 \mathrm{~ms}$, B) Learning trial 2: activation of P3 (in between $t>150$ to $t<=250 \mathrm{~ms}$ ) and activation of P1 (in between $t>350$ to $t<=450 \mathrm{~ms}$ ) as the results of pattern association.

After two trials, association of P1 $\leftrightarrow$ P3 could be established. Initially, intensified currents to subpopulations P1 and P3 activate their respective subpopulation only. Then, at times $350<t \leq 450 \mathrm{~ms}$, there exists prolonged activity of P1 by activation through P3. In trial 2, prospective activity can be observed with activation of P3 within stimulation period of P1 (in $150<t \leq 250 \mathrm{~ms}$ ) and activation of P1 within stimulation period of P3 (in $350<t \leq 450 \mathrm{~ms}$ ).

\subsection{Learning with Novel Stimuli}

For learning with novel stimuli, only $20 \%$ of neurons within the same subpopulation are initialised with $W_{1}$ values in the range of 0 and 1 . The initial values of $W_{1}$ represent some random connectivity assumed to result from any previous learning. Initially, in our simulation, the so intialised synaptic connections are not enough to have synchronous activity within a subpopulation compared to when learning with 
familiar stimuli. Results of association learning with novel stimuli P1 and P3 are depicted in Fig. 4.

A. Trial 1

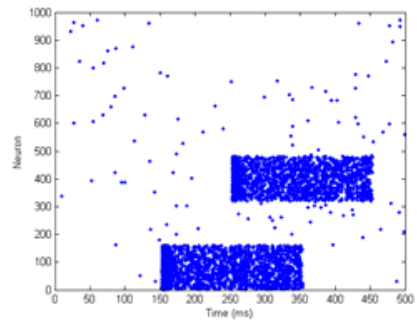

B. Trial 10

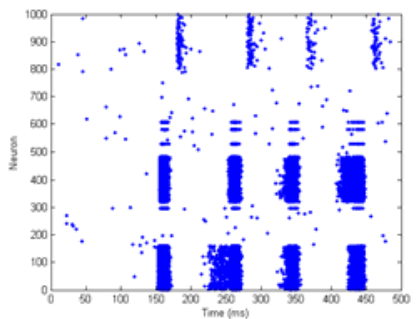

Fig. 4. Neuronal network activity after one and ten learning trials for stimuli pair P1 $\leftrightarrow$ P3. Currents to excitatory subpopulation neurons of P1 is intensified for $200 \mathrm{~ms}$ ( $t>150$ to $t<=350$ $m s)$, then P3 is stimulated for the same duration ( $t>250$ to $t<=450 \mathrm{~ms})$. A) In the early phase of learning, after one trial, neurons in subpopulations P1 and P3 fire asynchronously as both stimuli are novel and activity are only dependent on the external currents. B) After ten trials, neuronal activity within each subpopulation is more synchronised as the result of learning. Activation of P3 (within $t>150$ to $t<=250 \mathrm{~ms}$ ) and activation of P1 (within $t>400$ to $t<=500$ $m s$ ) indicate association of $\mathrm{P} 1 \leftrightarrow \mathrm{P} 3$.

From Fig. 4, during the early phase of learning, after stimulations to P1 and P3, the neurons in both subpopulations only fire asynchronously caused by the injected current within $t>150$ to $t \leq 350 \mathrm{~ms}$ and $t>250$ to $t \leq 450 \mathrm{~ms}$ for P1 and P3, respectively. A spill-over of activity from P1 to P3 and vise-versa can only be observed after ten trials.

\section{Conclusion}

We have explored a simple associative learning scheme utilising Hebbian learning both for spike rates and timings (STDP) for synaptic plasticity, similar to [9]. Unlike other supervised approaches [5] where neuronal activity is forced to have relatively precise spike timing to match the desired target spike train, this scheme uses supervisory currents to establish an association between two stimuli. And unlike previous approaches [8], that only rely on sliding average spike rates, our approach has a plausibility advantage by incorporating spike timings, too [9]. However, it remains to examine whether a single branch (1), (2) or (3) of the weight update rule has a dominating affect on learning. The associations show in spill-over of activity between the two stimuli involved. This demonstrates once more that long-term associations between stimuli involving synaptic plasticity are triggered by overlapping short-term activity involving only short-term activity dynamics. We have run a series of simulation experiments for learning associations of familiar stimuli and 
novel stimuli. For learning with familiar stimuli, associations between pair patterns are learned faster compared to novel stimuli.

Acknowledgments. This research has been funded by a $\mathrm{PhD}$ grant from the Ministry of Higher Education (Malaysia).

\section{References}

1. Bloom, F., Nelson, C. A., Lazerson, A.: Brain, Mind, and Behaviour (3rd ed.). Educational Broadcasting Corporation, US (2001)

2. Crick, F. (1989). The recent excitement about neural networks. Nature. 337, 129--132 (1989)

3. Izhikevich, E. M.: Simple Model of Spiking Neurons. IEEE Trans. Neural Networks. Vol. 14, No. 6, 1569--1572 (2003)

4. Izhikevich, E. M.: Which model to use for cortical spiking neurons. IEEE Trans. Neural Networks. Vol. 15, No. 5, 1063--1070 (2004)

5. Kasinski, A., Ponulak, F.: Comparison of Supervised Learning Methods for Spike Time Coding in Spiking Neural Networks. Int. J. Appl. Math. Comput. Sci. Vol. 16, No. 1, 101-113 (2006)

6. Dayan, P., Abbot, L. F.: Theoretical Neuroscience: Computational and Mathematical Modeling of Neural Systems. Cambridge, MA: MIT (2005).

7. Brunel, N., Lavigne, F.: Semantic Priming in a Cortical Network Model. Journal of Cognitive Neuroscience. Vol. 21, No. 12, 2300--2319 (2009)

8. Mongillo, G., Amit, D.J., Brunel, N.: Retrospective and prospective persistent activity induced by Hebbian learning in a recurrent cortical network. 18, 2011--2024 (2003)

9. Paugam-Moisy, H., Martinez, R., Bengio, S.: Delay learning and polychnization for reservoir computing. Neurocomputing. Vol. 71, Issue 7-9, 1143--1158 (2008) 